\title{
SUMS OF CANTOR SETS YIELDING AN INTERVAL
}

\section{CARLOS A. CABRELLI, KATHRYN E. HARE and URSULA M. MOLTER}

(Received 15 December 1998; revised 11 October 2001)

Communicated by A. H. Dooley

\begin{abstract}
In this paper we prove that if a Cantor set has ratios of dissection bounded away from zero, then there is a natural number $N$, such that its $N$-fold sum is an interval. Moreover, for each element $z$ of this interval, we explicitly construct the $N$ elements of $C$ whose sum yields $z$. We also extend a result of Mendes and Oliveira showing that when $s$ is irrational $C_{a}+C_{a^{s}}$ is an interval if and only if $a /(1-2 a) a^{s} /\left(1-2 a^{s}\right) \geq 1$.
\end{abstract}

2000 Mathematics subject classification: primary 28A80, 26A30.

Keywords and phrases: Cantor set, sums of sets.

\section{Introduction}

Cantor sets are sets that can be constructed in a similar fashion to the classical middle third Cantor set, but rather than using the ratio $1 / 3$ at each step, we allow the removed intervals to be variable in length and not necessarily centered.

Cantor sets appear in many different settings, and often one is interested in knowing about the arithmetic sum of two or more Cantor sets. For example, in number theory $N$-fold sums of Cantor sets have arisen in the study of continued fractions as initiated by Hall (see [6]). In harmonic analysis these sums have been studied by Brown et al. $([4,3])$ to aid with understanding the algebraic structure of the space of measures. In connection with the study of homoclinic tangencies in dynamical systems, Palis asked if the difference of two Cantor sets is either of Lebesgue measure zero or contains an interval [10, page 151]. This is false in full generality (see [2] or [12]) but very recently Moreira and Yoccoz [8] have shown that it is generically true for dynamically defined Cantor sets. 
Our primary interest is in understanding when the sum (or difference) of two or more Cantor sets contains an interval. In [5] this problem is completely solved for $N$-fold sums of central Cantor sets $C_{a}$ with fixed ratio of dissection $a$, and the solution implies Palis's conjecture is true for that case.

It appears to be much more difficult to characterize when the sum of two different, central Cantor sets, or $\mathrm{N}$-fold sums of more general Cantor sets, contain intervals. It was conjectured in [5] that if the ratios of a Cantor set are bounded away from zero then a sufficiently large $N$-fold sum is an interval. In this paper we prove that this conjecture is true, and moreover, give a constructive proof of it. The proof of our conjecture also yields a sufficient condition of when the sum of $N$ Cantor sets contains an interval.

We also continue the study of $C_{a}+C_{a^{s}}$, begun by Mendes and Oliveira in [7]. We prove that when $s$ is irrational $C_{a}+C_{a^{ \pm}}$is an interval if and only if the sufficient thickness condition from Newhouse's gap lemma ([9]) is met, answering an open problem left in [7]. We provide evidence to support the hypothesis that, in contrast, this sufficient condition is unnecessary for every rational number $s$; we reduce this problem to proving there is no rational solution to a certain equation.

\section{Definitions and notation}

By a Cantor set we mean a compact, totally disconnected, perfect subset of the reai line. The initial interval of a Cantor set $C$ is the closed interval of minimal length containing $C$. The gaps of a Cantor set $C$ are the bounded connected components of the complement of $C$. All Cantor sets can be constructed in a similar fashion to the classical middle third Cantor set. We begin with a compact interval and remove from it an open interval, leaving two closed intervals of positive length (to avoid isolated points) called the intervals of step one. The quotient between the length of these intervals and the initial interval are called the ratios of dissection at step one. A similar operation is performed on each interval of step one, producing the closed intervals (of positive length) of step two and the ratios of dissection at step two. This construction yields a decreasing sequence of closed sets whose intersection is a Cantor set when the union of the intervals removed is dense in the initial interval. Note that different constructions can yield the same Cantor set.

A central Cantor set is one in which the ratios of dissection at step $k$ are all the same, and hence a centred interval is removed from each interval of the previous step. We will denote by $C_{a}$ the central Cantor set with fixed ratio of dissection $a$; the classical middle third Cantor set is $C_{1 / 3}$.

Let $W$ denote the set of binary words of finite length:

$$
W=\{e\} \bigcup\left\{w_{1} \cdots w_{r}: w_{i} \in\{0,1\}, r \in \mathbb{N}\right\},
$$


where $\{e\}$ denotes the empty word. If $w, w^{\prime} \in W$ then $w w^{\prime}$ will denote the concatenation of $w$ and $w^{\prime}$, and the length of word $w$ will be denoted by $|w|$. Set $|e|=0$ and let $W^{*}$ denote the set of words of positive length. Given $w$, either an infinite binary word or a finite binary word of length at least $k$, we will denote by $w(k)$ the truncation $w_{1} \cdots w_{k}$.

It is convenient to use the elements of $W$ to describe the construction of a given Cantor set $C$. Let $I_{e}$ denote the initial (minimal) interval. If $w \in W,|w|=k$ and $I_{w}$ is an interval of step $k$ in the construction, then we denote by $I_{w 0}$ and $I_{w 1}$ the left and right intervals obtained by removing the open interval from $I_{w}$. The ratios of dissection of the Cantor set can similarly be labelled by $W^{*}$ in the natural way: $\left\{r_{w}:|w|=k\right\}$ are the ratios at step $k$. The initial interval and the ratios of dissection uniquely determine the construction of $C$.

For $w \in W,|w|=k$, set

$$
x[w] \equiv \begin{cases}r_{w(1)} \cdots r_{w(k-1)}\left(1-r_{w(k)}\right)\left|I_{e}\right| & \text { if } w_{k}=1 ; \\ 0 & \text { if } w_{k}=0 .\end{cases}
$$

The geometrical interpretation is that $x[w 1]$ is the sum of the length of the left subinterval and the gap of interval $I_{w}$. The interval $I_{w}$ has $\sum_{i=1}^{k} x[w(i)]$ as its left endpoint and has length $\left|I_{w}\right|=x[w 1]+x[w 11]+\cdots$.

Each $c \in C$ can be labelled by the (unique) infinite binary word $w$ which has the property that $c \in I_{w(k)}$ for each truncation $w(k)$ of $w$. Since the lengths of the intervals $I_{w(k)}$ tend to zero as $k \rightarrow \infty, c$ is the limit of the left endpoints of these intervals, and therefore $c=\sum_{i=1}^{\infty} x[w(i)]$. Conversely, as $C$ is closed, $\sum_{i=1}^{\infty} x[w(i)] \in C$, and hence there is a 1-1 correspondence between $C$ and the set of infinite binary words.

\section{Cantor sets with bounded ratios of dissection}

In this section we will prove that for any positive number $a$ there exists an integer $n$ such that the sum of $n$ arbitrary Cantor sets with ratios of dissection greater than $a$ contains an interval. An immediate corollary of this is that if a Cantor set has rates bounded away from zero, then the sum of enough copies of itself will contain an interval.

The proof is constructive and provides a method to select for each element $z$ in an appropriate interval, elements, one from each Cantor set, whose sum is $z$.

We first need to prove the following rather technical lemma.

LEMMA 3.1. Let $C$ be a Cantor set with initial interval $I_{e}$ and ratios of dissection $\left\{r_{w}: w \in W^{*}\right\}$. Suppose there is some $0<a \leq 1 / 3$ such that $r_{w} \geq$ a for all $w \in W^{*}$. 
(i) If $w, v \in W$ then

$$
x[w v 1] \frac{a}{(1-a)^{2}} \leq x[w 1] .
$$

(ii) If $w \in W$, then the length of either the left or the right subinterval of $I_{w}$ is at least $(a /(1-a)) \times[w 1]$, that is,

$$
\left|I_{w 0}\right| \geq\left(\frac{a}{1-a}\right) x[w 1], \quad\left|I_{w 1}\right| \geq\left(\frac{a}{1-a}\right) x[w 1] .
$$

ProOF. (i) Note that since $a \leq 1 / 3$ we have $a /(1-a)^{2} \leq 1$, thus if $v=e$ the result is clear.

So assume $|w|=k$ and $|v|=j>0$. By definition

$$
\begin{aligned}
x[w v 1] & =r_{w(1)} \cdots r_{w(k)} r_{w v(k+1)} \cdots r_{w v(k+j)}\left(1-r_{w v(k+j) 1}\right)\left|I_{e}\right| \\
& =x[w 1]\left(\frac{1}{1-r_{w 1}}\right) r_{w v(k+1)} \cdots r_{w v(k+j)}\left(1-r_{w v(k+j) 1}\right) .
\end{aligned}
$$

Since all ratios of dissection are at least $a$, all ratios are also at most $1-a$, and hence the result follows directly.

(ii) Because $x[w 1]$ is the sum of the length of the left subinterval and the gap of $I_{w}$, this is an easy consequence of all ratios being at least $a$.

Now we can state and prove the main theorem.

THEOREM 3.2. Suppose $C_{1}, \ldots, C_{n}$ are Cantor sets which have constructions with ratios of dissection all at least $a$, where $0<a \leq 1 / 3$. If $n \in \mathbb{N}$ is such that

$$
(n-1) \frac{a^{2}}{(1-a)^{3}}+\frac{a}{1-a} \geq 1
$$

then $C_{1}+C_{2}+\cdots+C_{n}$ contains an interval.

If, in addition, no translate of any one of these Cantor sets is contained in a gap of another, then $C_{1}+C_{2}+\cdots+C_{n}=[A, B]$, where $A=\min \left\{\sum_{i=1}^{n} c_{i}: c_{i} \in C_{i}\right\}$ and $B=\max \left\{\sum_{i=1}^{n} c_{i}: c_{i} \in C_{i}\right\}$.

PROOF. We will assume, without loss of generality, that the Cantor sets $C_{i}$ have initial intervals $I_{e}^{(i)}=\left[0, b_{i}\right]$ and ratios of dissection $\left\{r_{w}^{(i)}: w \in W^{*}\right\}$ with $r_{w}^{(i)} \geq a$ for all $i=1, \ldots, n$ and $w \in W^{*}$.

We will prove that the sum $C_{1}+C_{2}+\cdots+C_{n}$ contains the interval $\left[0, \min _{i} b_{i}\right]$ by showing that if $z \in\left[0, \min _{i} b_{i}\right]$, then we can choose $n$ infinite binary words $w^{(i)}$, $i=1, \ldots, n$, such that

$$
z \in \bigcap_{k}\left(I_{w^{(1)}(k)}^{(1)}+\cdots+I_{w^{(n)}(k)}^{(n)}\right)
$$


or equivalently, $z=c_{1}+c_{2}+\cdots+c_{n}$, for $c_{i}=\sum_{k=1}^{\infty} x^{(i)}\left[w^{(i)}(k)\right] \in C_{i}$. (For notational ease we will simply write $x\left[w^{(i)}(k)\right]$ for $x^{(i)}\left[w^{(i)}(k)\right]$ in what follows.)

If $z=0$ or $z=\min b_{i}$ this is trivial, take either all $w_{k}^{(i)}=0$ for all $i$, or all $w_{k}^{(j)}=0$ for $j \neq i$ and $w_{k}^{(i)}=1$ respectively, where $i$ is the index that yields the minimum interval.

So assume otherwise. We will inductively construct the words $w^{(i)}$, defining one digit, of one of the sequences, at each step in the process. To begin, choose the index $i$ such that $x^{(i)}[1]$ is maximal (that is, the sum of the length of the left subinterval and the gap of $I_{e}^{(i)}$ is largest). If $x^{(i)}[1]>z$, then we can only have $z=\sum c_{j}$ if $c_{i} \in I_{0}^{(i)}$, thus we must define $w_{1}^{(i)}=0$. Otherwise, $x^{(i)}[1] \leq z$. Then $z \in \sum_{j} I_{w^{(j)}}^{(j)}$, where $w^{(j)}=e$ if $j \neq i$ and $w^{(i)}=1$. Thus we will choose to define $w_{1}^{(i)}=1$. Set $y_{1}=0$ or $x^{(i)}[1]$ respectively. (In either case $y_{1}=x\left[w_{1}^{(i)}\right]$.) Clearly, $y_{1} \in C_{1}+\cdots+C_{n}$ and $y_{1} \leq z$.

Now assume that at step $m$ we have chosen the first $m_{i}$ digits of $w^{(i)}$, for $i=$ $1, \ldots, n$, where $\sum_{i=1}^{n} m_{i}=m$. Each finite word $w^{(i)}\left(m_{i}\right)$ specifies an interval in step $m_{i}$ of the construction of $C_{i}$ whose left endpoint is given by $\sum_{k=1}^{m_{i}} x\left[w^{(i)}(k)\right]$. Assume $y_{m} \in C_{1}+\cdots+C_{n}$ is the sum of these endpoints,

$$
y_{m}=\sum_{i=1}^{n} \sum_{k=1}^{m_{i}} x\left[w^{(i)}(k)\right],
$$

and that $z_{m}=z-y_{m} \geq 0$.

If $z_{m}=0\left(z=y_{m}\right)$, we quit and complete all $w^{(i)}$ with 0 's. In this case we clearly have $z=\sum_{i=1}^{n} \sum_{k=1}^{\infty} x\left[w^{(i)}(k)\right]$ as we desired to show. Otherwise we will construct $y_{m+1}$. For this we first need to pick the index $i=i(m)$ which determines the word $w^{(i)}$ to be modified. To accomplish this, choose $i$ such that the length of the left subinterval and gap of $I_{w^{(i)}\left(m_{i}\right)}^{(i)}$ is maximal, that is,

$$
x\left[w^{(i)}\left(m_{i}\right) 1\right] \geq x\left[w^{(j)}\left(m_{j}\right) 1\right], \quad \text { for } j=1, \ldots, n .
$$

(We interpret $w^{j}(0)=e$.) Having chosen this index $i$, we then define

$$
w_{m_{i}+1}^{(i)}= \begin{cases}0 & \text { if } x\left[w^{(i)}\left(m_{i}\right) 1\right]>z_{m} \\ 1 & \text { otherwise. }\end{cases}
$$

In other words, at step $m+1$ we modify the sequence corresponding to (one of) the largest value(s) of $x\left[w^{(i)}\left(m_{i}\right) 1\right]$. If adding the segment $x\left[w^{(i)}\left(m_{i}\right) 1\right]$ to $y_{m}$ is still smaller than $z$, we increase $y_{m}$ by that amount, and otherwise set $y_{m+1}=y_{m}$. In either case, $y_{m+1}=y_{m}+x\left[w^{(i)}\left(m_{i}+1\right)\right]$ is the sum of the left endpoints of the intervals $I_{w^{(i)}\left(m_{i}+1\right)}^{(i)}$ and $I_{w^{(j)}\left(m_{j}\right)}^{(j)}$, for $j \neq i$. This seems a reasonable way to proceed, at least naively, because if $z \in \sum_{j}\left(I_{\left.w^{j}\right)\left(m_{j}\right)}^{(j)} \cap C_{j}\right)$, then $z \in \sum_{j \neq i} I_{w^{(j)}\left(m_{j}\right)}^{(j)}+I_{w^{(i)}\left(m_{i}+1\right)}^{(i)}$. 
(The main content of the proof is in showing that this "reasonable" approach actually works.)

We should remark that if this process doesn't stop (with $y_{m}=z$ ) then all letters of the infinite binary words $w^{(i)}$ are eventually determined because the fact that $x\left[w^{(i)}(n)\right] \rightarrow 0$ as $n \rightarrow \infty$ ensures that every index $i$ is chosen to be modified infinitely often.

We will prove that $z=\sum_{i=1}^{n} \sum_{k=1}^{\infty} x\left[w^{(i)}(k)\right]$, or, equivalently, verify that $y_{m} \rightarrow z$. To show this we claim it suffices to prove that at least one of the infinite binary words $w^{(i)}$ has infinitely many 0 's. To see why this is sufficient suppose $z_{m} \geq \varepsilon>0$ for all $m$ (recall that $z_{m}=z-y_{m}$ is a decreasing sequence) and that the letter 0 occurs infinitely often in $w^{(i)}$. If $w_{k}^{(i)}=0$, then by construction this means that $x\left[w^{(i)}(k-1) 1\right]>z_{m(k)} \geq \varepsilon$ where $m(k)$ is the step on which $w_{k}^{(i)}$ was defined. But $x\left[w^{(i)}(k)\right] \rightarrow 0$ as $k \rightarrow \infty$, which gives a contradiction.

So it only remains to show that there is some index with infinitely many zeroes. We proceed by contradiction. Assume each of the $w^{(i)}$ has only a finite number of zeroes. Let $m=m_{1}+\cdots+m_{n}$ be the step where the last zero was chosen and assume (without loss of generality) that $w_{m_{n}}^{(n)}$ was the last zero.

Note that the sequence $\left\{y_{M}\right\}$ is non-decreasing and bounded above by $z$, thus

$$
\lim _{M \rightarrow \infty} y_{M} \leq z
$$

Since only l's are chosen after step $m$, we have $\lim _{M} y_{M}=y_{m}+\sum_{j=1}^{n}\left|I_{w^{\prime}\left(m_{j}\right)}^{(j)}\right|$, and because zero was chosen at step $m$ we have that $y_{m}=y_{m-1}$. Hence

$$
\lim _{\mathcal{M}} y_{M}=y_{m-1}+\sum_{j=1}^{n-1}\left|I_{w^{j}\left(m_{j}\right)}^{(j)}\right|+\left|I_{w^{n}\left(m_{n}-1\right) 0}^{(n)}\right| .
$$

But by the second part of Lemma 3.1 this yields directly that

$$
\lim _{M} y_{M} \geq y_{m-1}+\frac{a}{1-a}\left(\sum_{j=1}^{n-1} x\left[w^{(j)}\left(m_{j}-1\right) 1\right]+x\left[w^{(n)}\left(m_{n}-1\right) 1\right]\right) \text {. }
$$

Since $z<\left|I_{e}^{(j)}\right|$ for all $j$, it is clear that

$$
m_{j} \geq 1 \text { for } 1 \leq j \leq n .
$$

Thus each index must have been chosen at least once before the final zero at step $m$. Hence, if $j<n$, then $w_{m_{j}}^{(j)}$ was determined in an earlier step than $m$, and that could only have occurred if there were integers $k_{j}<m_{n}$ such that

$$
x\left[w^{(j)}\left(m_{j}-1\right) 1\right] \geq x\left[w^{(n)}\left(k_{j}\right) 1\right] .
$$


By the first part of the lemma

$$
x\left[w^{(n)}\left(k_{j}\right) 1\right] \geq \frac{a}{(1-a)^{2}} x\left[w^{(n)}\left(m_{n}-1\right) 1\right],
$$

thus, considering equations (3), (5) and (6) we have that

$$
\lim _{M} y_{M} \geq y_{m-1}+x\left[w^{(n)}\left(m_{n}-1\right) 1\right]\left(\frac{a}{1-a}+\frac{a^{2}}{(1-a)^{3}}(n-1)\right) .
$$

The choice of $n$ yields $\lim _{M} y_{M} \geq y_{m-1}+x\left[w^{(n)}\left(m_{n}-1\right) 1\right]$. This leads to a contradiction since the fact that $w^{(n)}\left(m_{n}\right)=0$ means that $x\left[w^{(n)}\left(m_{n}-1\right) 1\right]$ is strictly bigger than $z_{m-1}=z-y_{m-1}$ or, equivalently,

$$
\lim _{M} y_{M} \geq y_{m-1}+x\left[w^{(n)}\left(m_{n}-1\right) 1\right]>z,
$$

which contradicts (1). Thus there must be infinitely many zeroes, and this completes the proof of the first part of the theorem.

For the second part, $z$ is chosen arbitrarily in $[A, B]$. The same proof can be carried out, except that we need to justify why we still have (4). We proceed by contradiction: suppose there exists some index $j$ such that $m_{j}=0$, that is, the index $j$ was never chosen to be the modified index before the last zero was specified. This means that $w_{k}^{(j)}=1$ for all $k$. Therefore, using (2), we have

$$
z \geq \lim _{M} y_{M} \geq y_{m-1}+\left|I_{e}^{(j)}\right|+\left|I_{w^{n}\left(m_{n}\right)}^{(n)}\right| \text {. }
$$

Geometrically this says $z$ is at least $y_{m-1}$ plus the length of the left subinterval of $I_{w^{n}\left(m_{n}-1\right)}^{(r)}$ (recall the word $w^{(n)}\left(m_{n}\right)$ ends in 0 ) and the length of the initial interval of the Cantor set $C_{j}$. But again we use the fact that $w_{m_{n}}^{(n)}=0$ means that $z<$ $x\left[w^{(n)}\left(m_{n}-1\right) 1\right]+y_{m-1}$. Another way to say this is that $z$ is less than $y_{m-1}$ plus the left subinterval and gap of $I_{w^{n}\left(m_{n}-1\right)}^{(n)}$. Since (by the additional hypothesis) no translate of $C_{j}$ is contained in any gap of $C_{n}$ it follows that the length of $I_{e}^{(j)}$ is at least the length of the gap of $I_{w^{n}\left(m_{n}-1\right)}^{(n)}$ and this gives a contradiction. Thus each index must have been chosen at least once before the nal zero, so each $m_{j} \geq 1$ and this completes the proof of the theorem.

The reader may have noticed that for certain Cantor sets the method of proof can be modified to yield better results. We give two examples below.

COROLlaRY 3.3. Suppose $C_{1}, \ldots, C_{n}$ are central Cantor sets with initial intervals $I_{e}^{(i)}$ and ratios of dissection $r_{w}^{(i)} \geq a_{i}$ for $i=1, \ldots, n$. If $\sum_{i=1}^{n}\left(a_{i} /\left(1-a_{i}\right)\right) \geq 1$, then $C_{1}+\cdots+C_{n}$ contains an interval.

If, in addition, no translate of any one of these sets $C_{i}$ is contained in a gap of another then $C_{1}+\cdots+C_{n}=I_{e}^{(1)}+\cdots+I_{e}^{(n)}$. 
PROOF. The length of the intervals in the construction of a central Cantor set decrease monotonically with the step of the construction. Thus $x^{(i)}[w v 1] \leq x^{(i)}[w 1]$ for all $w, v \in W$ and $i=1, \ldots, n$. From the lemma we know that the length of both the left and right subinterval of $I_{w(k)}^{(i)}$ is at least $\left(a_{i} /\left(1-a_{i}\right)\right) x^{(i)}[w(k) 1]$. The corollary easily follows by using these two observations in the proof of the theorem instead of the lemma.

REMARK. This result is best possible when all $C_{i}=C$ (see [5]).

COROLlaRY 3.4. Let $C_{a b}$ denote the Cantor set in $[0,1]$ with all left ratios of dissection equal to $a$ and right ratios equal to $b$. Suppose

$$
(N-1) \min \{a, b\} \geq 1-a-b .
$$

Then $(N) C_{a b}=[0, N]$.

ProOF. Without loss of generality we may assume $a \leq b$ for otherwise we reverse the roles of left and right endpoints and intervals. We claim that for such Cantor sets one has $x[w v 1] \leq x[w 1]$. This is certainly clear if $v=e$ or if $v_{1}=0$. If, instead, $v_{1}=\cdots=v_{k}=1$, then $x[w v 1] \leq\left|I_{w}\right| b^{k}(1-b)$, while $x[w 1]=\left|I_{w}\right|(1-b)$. Also,

$$
\left|I_{w(k) 1}\right| \geq\left|I_{w(k) 0}\right|=a\left|I_{w(k)}\right|=\frac{a}{1-b} x[w(k) 1] .
$$

Using these estimates in place of the lemma we obtain the result.

REMARK. This estimate is sharper than the one obtained in [5], and Corollary 4.3 below demonstrates that this choice of $N$ is also best possible to get the whole interval.

\section{The sum $C_{a}+C_{b}$}

In this section we will concentrate on the study of sums of two different central Cantor sets continuing the investigation began by Mendes and Oliveira in [7].

Here the notion of thickness will play a fundamental role. We define this as in [10]: Given a gap $G$ of a Cantor set $C$ a bridge $B$ of $G$ is a maximal interval whose boundary intersects the boundary of $G$ and which contains no point of a gap whose length is at least the length of $G$. We refer to $(B, G)$ as a bridge/gap pair. The thickness of the Cantor set $C$ is defined as

$$
\tau(C) \equiv \inf \left\{\frac{|B|}{|G|}:(B, G) \text { a bridge/gap pair }\right\} .
$$

The Newhouse gap lemma [9] says that if $C_{1}$ and $C_{2}$ are Cantor sets, and the product of their thicknesses is at least one, then $C_{1}+C_{2}$ contains an interval. The proof given in $[10,4.2]$ actually shows more, however, namely: 
Proposition 4.1. Let $C_{1}$ and $C_{2}$ be Cantor sets such that whenever $\left(B_{i}, G_{i}\right)$ is a bridge/gap pair of $C_{i}$ for $i=1,2$ then either $\left|B_{1}\right| \geq\left|G_{2}\right|$ or $\left|B_{2}\right| \geq\left|G_{1}\right|$. Then $C_{1}+C_{2}$ contains an interval. If moreover, none of the translates of either of the Cantor sets are contained in a gap of the other, then $C_{1}+C_{2}$ is an interval.

Before proving the main theorem of this section, we need first the following simple, but useful, result which demonstrates a sense in which the bridge/gap condition is necessary. Denote by $\mathbb{R}^{+}$the set of positive real numbers.

Proposition 4.2. Suppose $C_{1}, C_{2}, \ldots, C_{n}$ are Cantor sets in $\mathbb{R}^{+}$and $I_{i}=\left(a_{i 1}, a_{i 2}\right)$ are intervals such that $I_{i}$ is included in a gap of $C_{i}$ for each $i=1, \ldots, n$. Let $a_{2}=\min a_{i 2}$. If $a_{2}>\sum_{j=1}^{n} a_{j 1}$ then $C_{1}+\cdots+C_{n}$ has gaps. In particular,

$$
\left(C_{1}+\cdots+C_{n}\right) \bigcap\left(\sum_{j=1}^{n} a_{j 1}, a_{2}\right)
$$

is empty.

PRoOF. Choose $x \in\left(\sum_{j=1}^{n} a_{j 1}, a_{2}\right)$ and assume $x=\sum b_{i}, b_{i} \in C_{i}$. Since $x<a_{i 2}$ for all $i$ we must have $b_{i}<a_{i 2}$. As $b_{i} \in C_{i}$ this implies $b_{i} \leq a_{i 1}$. But then $x \leq \sum_{j=1}^{n} a_{j 1}$ which is obviously a contradiction.

COROLLARY 4.3. If $C$ has gap $(a, b)$ with $b-a>(N-1) a$ then $(N) C$ is not an interval.

Let us now state and prove our main result of this section.

THEOREM 4.4. Let $C_{a}$ and $C_{a^{s}}$ be central Cantor sets, with initial interval $[0,1]$, with ratios of dissection $a$ and $a^{s}$ respectively, $a<1 / 2$ and $s>1$. The following are equivalent:

(1) $(i) C_{a}+(j) C_{a^{s}}=[0, i+j]$.

(2) For every $m, n \in \mathbb{N}$ at least one of the following inequalities holds:

$$
\begin{aligned}
a^{s(n-1)}\left(1-(j+1) a^{s}\right) & \leq i a^{m}, \\
a^{m-1}(1-(i+1) a) & \leq j a^{s n} .
\end{aligned}
$$

(3) There are no positive integers $n, m$ such that

$$
\frac{\log ((1-(i+1) a) / j)}{\log a}-s-1<n s-m<\frac{\log \left(i /\left(1-(j+1) a^{s}\right)\right)}{\log a} .
$$

REMARK. The case $s=1$ was done in [5]. 
ProOF. First observe that if $C_{b}$ is a Cantor set of ratio of dissection $b$ with initial interval $[0,1]$, then $C_{b}$ has gaps of size $b^{n-1}(1-2 b)$ with corresponding bridges of length $b^{n}$.

In [5] it was shown that if $k$ satisfies that $1 /(k+1) \leq b<1 / k$, and $q<k$, then $(q) C_{b}$ is a Cantor set, and for $q \geq k,(q) C_{b}=[0, q]$. For values of $q$ smaller than $k$ it is easy to verify that $(q) C_{b}$ has gaps of size $b^{n-1}(1-(q+1) b)$, with bridges of length $q b^{n}$, for some integer $n$. Also, the intervals $\left(q b^{n}, b^{n-1}(1-b)\right)$ are gaps of $(q) C_{b}$ for all $n$.

(1) implies (2). If (i) $C_{a}=[0, i]$ then $a \geq 1 /(i+1)$, so inequality (b) is satisfied. A similar argument shows that if $(j) C_{a^{s}}=[0, j]$, then (a) is satisfied.

We will now prove the implication for the case when $(i) C_{a}$ and $(j) C_{a^{s}}$ are Cantor sets. If both inequalities (a) and (b) fail for some $m, n \in \mathbb{N}$ then

$$
a^{s(n-1)}\left(1-a^{s}\right)>i a^{m}+j a^{s n}
$$

and

$$
a^{m-1}(1-a)>j a^{s n}+i a^{m} .
$$

We can apply Proposition 4.2 to the Cantor sets, $(i) C_{a}$ and $(j) C_{a^{s}}$, and their gaps, $\left(i a^{m}, a^{m-1}(1-a)\right)$ and $\left(j a^{n s}, a^{(n-1) s}\left(1-a^{s}\right)\right)$, since assumptions (7) and (8) imply that the hypothesis of Proposition 4.2 is satisfied. Thus $(i) C_{a}+(j) C_{a^{s}}$ has a gap and this contradicts (1).

(2) implies (1). If (i) $C_{a}$ and $(j) C_{a^{s}}$ are Cantor sets, then inequalities (a) and (b) are exactly the conditions imposed on the bridge-gap pairs in Proposition 4.1 for the Cantor sets $(j) C_{a^{s}}$ and $(i) C_{a}$. Since $C_{a}$ and $C_{a^{s}}$ have initial intervals [0,1], it is easy to see that the translation condition is also satisfied for $(i) C_{a}$ and $(j) C_{a^{3}}$. So we conclude that its sum is the interval $[0, i+j]$.

(2) if and only if (3). This is simple algebra.

An interesting special case is when $s$ is irrational.

COROLLARY 4.5. If $s$ is irrational, then $(i) C_{a}+(j) C_{a^{s}}=[0, i+j]$ if and only if the product of the thicknesses of $(i) C_{a}$ and $(j) C_{a^{s}}$ is at least one.

ProOF. We only need to prove necessity when $s \notin \mathbb{Q}$ since sufficiency is true for all $s$ by Newhouse's lemma. So assume the product of the thicknesses is less than one. As $\tau\left((k) C_{b}\right)=k b /(1-(k+1) b)$, this implies that

$$
L \equiv \frac{\log ((1-(i+1) a) / j)}{\log a}-s-1<\frac{\log \left(i /\left(1-(j+1) a^{s}\right)\right)}{\log a} \equiv R .
$$

When $s$ is not rational the semigroup $\{n s \bmod 1: n \in \mathbb{N}\}$ is dense in $[0,1]$ and consequently there are infinitely many positive integer pairs, $n, m$, such that $n s-m \in$ 
$[L, R]$. Thus condition (3) of the theorem fails (infinitely often) so that not only is (i) $C_{a}+(j) C_{a^{s}} \neq[0, i+j]$, but in fact there are gaps in the sum accumulating to zero.

Another interesting case is when $s=q$ or $1+1 / q$ for some integer $q$. In [7] Mendes and Oliveira prove that if $a^{s}=1-2 a$ then $C_{a}+C_{a^{2}}=[0,2]$. Our theorem can be used to prove the converse.

COROLlARY 4.6. Suppose $a^{s}=1-2$ a for some $s>1$. Then $C_{a}+C_{a^{s}}=[0,2]$ if and only if $s=q$ or $1+1 / q$ for some positive integer $q$.

PROOF. Sufficience was proved in [7], but since it follows so easily from our theorem, we give a new proof here as well as the proof of necessity.

When $a^{s}=1-2 a$, condition (3) of the theorem implies that $C_{a}+C_{a^{s}}=[0,2]$ if and only if there are no positive integers $n, m$ such that

$$
-1<n s-m<-\frac{\log (4 a-1)}{\log a} .
$$

Note that as $a<1 / 2,-\log (4 a-1) / \log a<0$. Consequently, if $s$ is an integer, then there are clearly no integers $n, m$ so that (9) holds, that is, $C_{a}+C_{a^{s}}=[0,2]$. If, instead, $s=1+1 / q$ for some integer $q>1$, then the first element of the form $n s-m$ bigger than -1 is $-1+1 / q$. Hence it is enough to show that

$$
-\frac{\log (4 a-1)}{\log a}<-1+\frac{1}{q} \text { or, equivalently, } 4 a-1>a^{1-1 / q} .
$$

The assumptions $a^{s}=1-2 a$ and $s>1$ imply that $a>1 / 3$, while $s=1+1 / q$ implies $a^{1-1 / q}=a^{2} /(1-2 a)$. It follows after some calculations that $4 a-1<a^{1-1 / q}$, and thus (9) again fails to hold for any integers $n, m$.

To prove necessity notice first that if $a^{s}=1-2 a$ for some $s>1$, then $\tau\left(C_{a}\right) \tau\left(C_{a^{s}}\right)<1$. Corollary 4.5 implies that if $C_{a}+C_{a^{s}}=[0,2]$ then $s$ cannot be irrational. Thus we may assume $s=z+p / q$, with positive integers $z, p, q$ satisfying $p<q, \operatorname{gcd}(p, q)=\mathbf{Y}$ and either $z \geq 2$ or $p \geq 2$.

Let $a(s)$ denote the unique solution to $a^{s}=1-2 a$. Clearly $a(s)$ increases (to $\left.1 / 2\right)$ as $s$ increases, and since $s \geq 1+2 / q, a(s) \geq a(1+2 / q)$. Set $f(x)=4 x-1-x^{1-1 / q}$. If $x>1 / 3$, then

$$
f^{\prime}(x)=4-(1-1 / \dot{q}) x^{-1 / q} \geq 4-(1-1 / q)(1 / 3)^{-1 / q}>0,
$$

so $f(x)$ is an increasing function. It can be seen that $f(a(1+2 / q))>0$, and therefore $f(a(s))>0$ for all $s=z+p / q$, with $z \geq 2$ or $p \geq 2$. Hence $4 a(s)-1>(a(s))^{1-1 / q}$. Choosing integers $n$ and $m$ such that $n s-m=-1+1 / q$ we obtain a violation of condition (9). This completes the proof. 
In view of Corollary 4.5, it is of interest that there actually exist Cantor sets $C_{a}$ and $C_{a^{s}}$ such that $\tau\left(C_{a}\right) \tau\left(C_{a^{s}}\right)<1$ and $C_{a}+C_{a^{s}}=[0,2]$. In fact, we speculate that for every rational number $s$, there is a choice of $a$ satisfying $\tau\left(C_{a}\right) \tau\left(C_{a^{s}}\right)<1$, but having $C_{a}+C_{a^{s}}=[0,2]$.

We have partial results that support this claim.

Proposition 4.7. Let $s=1+p / q$, where $\operatorname{gcd}(p, q)=1$ and $p=1,2, \ldots, 8$, or let $s=n+1 / 2, n \in \mathbb{N}$. Then $C_{a}+C_{a^{s}}=[0,2]$ for some a $<t$ where $t=t(s)$ is the unique solution to $\tau\left(C_{t}\right) \tau\left(C_{t^{s}}\right)=1$.

The proof of this proposition relies on a rather technical proposition which is again a consequence of the theorem.

Proposition 4.8. Suppose $s=n+p / q$ where $n, p, q \in \mathbb{N}$ and $\operatorname{gcd}(p, q)=1$. Let $t=t(s)$ be the unique solution to $\tau\left(C_{t}\right) \tau\left(C_{t^{s}}\right)=1$. There is some $a<t$ such that $C_{a}+C_{a^{s}}=[0,2]$ if and only if there is no integer $r$ with $t^{r / q}=1-2 t^{s}$.

PROOF. Both directions of the proof will use the following calculation: If $t$ satisfies $\tau\left(C_{t}\right) \tau\left(C_{t}\right)=1$, then

$$
-\frac{\log \left(1-2 t^{s}\right)}{\log t}=\frac{\log (1-2 t)}{\log t}-s-1 .
$$

First, suppose that there exists $r$ such that $t^{r / q}=1-2 t^{s}$ or, equivalently,

$$
\frac{r}{q}=\frac{\log \left(1-2 t^{s}\right)}{\log t}
$$

As both $\log (1-2 x) / \log x$ and $\log \left(1-2 x^{s}\right) / \log x$ are increasing functions for $x<1 / 2$, when $a<t$ we must have

$$
\begin{aligned}
\frac{\log (1-2 a)}{\log a}-s-1 & <\frac{\log (1-2 t)}{\log t}-s-1=\frac{\log \left(1-2 t^{s}\right)}{\log t} \\
& =-\frac{r}{q}<-\frac{\log \left(1-2 a^{s}\right)}{\log a} .
\end{aligned}
$$

Since $\operatorname{gcd}(p, q)=1$ there are certainly positive integers $n, m$ such that $n s-m=$ $-r / q$. Thus condition (3) of the theorem implies $C_{a}+C_{a^{s}} \neq[0,2]$.

Assume now that there does not exist any $r$ satisfying the above condition. Choose the unique integer $r$ such that $t^{r / q}<1-2 t^{s}<t^{(r-1) / q}$. Then

$$
-\frac{r}{q}<\frac{\log (1-2 t)}{\log t}-s-1=-\frac{\log \left(1-2 t^{s}\right)}{\log t}<-\frac{r-1}{q} .
$$


Choose $a<t$ (but sufficiently close) so that the inequalities still hold, that is,

$$
-\frac{r}{q} \leq \frac{\log (1-2 a)}{\log a}-s-1<-\frac{\log \left(1-2 a^{s}\right)}{\log a} \leq-\frac{r-1}{q} .
$$

Since $n s-m$ is of the form $z / q$ for some integer $z$, equation (11) ensures we cannot have

$$
\frac{\log (1-2 a)}{\log a}-s-1<n s-m<-\frac{\log \left(1-2 a^{s}\right)}{\log a}
$$

for any $n, m \in \mathbb{N}$, and consequently $C_{a}+C_{a^{s}}=[0,2]$.

REMARK. Since (11) is obviously necessary as well as sufficient, the proof shows that the minimal $a$ such that $C_{a}+C_{a^{s}}=[0,2]$ is the maximum of the solutions to the equations

$$
-\frac{r}{q}=\frac{\log (1-2 x)}{\log x}-s-1 \text { and } \frac{r-1}{q}=\frac{\log \left(1-2 x^{s}\right)}{\log x} .
$$

We can now prove Proposition 4.7. The proof is straightforward, but rather technical, so we will only outline its main steps.

PROOF. Since $t$ satisfies the thickness condition and $s>1$, we have $1 / 3<t<1 / 2$. Also, $t$ satisfies the equations

$$
(1-2 t)\left(1-2 t^{s}\right)=t^{s+1} \text { and } t^{s}=(1-2 t) /(2-3 t) .
$$

Thus $t$ solves $t^{r / q}=1-2 t^{s}$ if and only if $t^{1-r / q}=2-3 t$.

By the previous proposition, it is therefore enough to show that this cannot occur for any of the choices of $s$ above. We leave this verification to the reader.

\section{Acknowledgements}

The authors thank S. Astels for sharing his results [1] before publication. His generalization of the Newhouse gap lemma leads to an alternative (non-constructive) proof of the fact that the sum of sufficiently many copies of a Cantor set with ratios of dissection bounded away from zero contains an interval.

\section{References}

[1] S. Astels, 'Cantor sets and numbers with restricted partial quotients', Trans. Amer. Math. Soc. 352 (2000), 133-170. 
[2] R. Bamon, S. Plaza and J. Vera, 'On central Cantor sets with self-arithmetic difference of positive Lebesgue measure', J. London Math. Soc. 52 (1995), 137-146.

[3] G. Brown, M. Keane, W. Moran and E. Pearce, 'An inequality, with applications to Cantor measures and normal numbers', Mathematika 35 (1988), 87-94.

[4] G. Brown and W. Moran, 'Raikov systems and radicals in convolution measure algebras', J. London Math. Soc. 28 (1983), 531-542.

[5] C. Cabrelli, K. Hare and U. Molter, 'Sums of Cantor sets', Ergodic Theory Dynam. Systems 17 (1997), 1299-1313.

[6] M. Hall, Jr., 'On the sum and product of continued fractions', Ann. of Math. (2) 48 (1947), 966-993.

[7] P. Mendes and F. Oliveira, 'On the topological structure of the arithmetic sum of two Cantor sets', Nonlinearity 7 (1994), 329-343.

[8] C. Moreira and J. Yoccoz, 'Stable intersections of Cantor sets with large Hausdorff dimension', Ann. of Math. (2) 154 (2001), 45-96.

[9] S. Newhouse, 'Lectures on dynamical systems', in: Dynamical systems, CIME Lectures, Bressanone, Italy, 1978, Progress in Math. 8 (Birkhauser, Boston, Mass., 1980) pp. 1-114.

[10] J. Palis and F. Takens, Hyperbolicity and sensitive chaotic dynamics at homoclinic bifurcations, Cambridge Stud. Adv. Math. 35 (Cambridge University Press, Cambridge, 1993).

[11] R. Salem, 'On sets of multiplicity for trigonometric series', Amer. J. Math. 64 (1942), 531-538.

[12] A. Sannami, 'An example of a regular Cantor set whose difference set is a Cantor set with positive measure', Hokkaido Math. J. 21 (1992), 7-24.

\section{CONICET and}

Departamento de Matematica

FCEyN, Universidad de Buenos Aires

Cdad. Universitaria, Pab. I

(1428) Bs.As.

Argentina

e-mail: ccabrelli@dm.uba.ar
Department of Pure Mathematics

University of Waterloo

Waterloo, Ont. N2L 3G1

Canada

e-mail: kehare@math.uwaterloo.ca

\section{CONICET and}

\section{Departamento de Matematica}

FCEyN, Universidad de Buenos Aires

Cdad. Universitaria, Pab. I

(1428) Bs.As.

\section{Argentina}

e-mail: umolter@dm.uba.ar 